\title{
Caracterização Eletromagnética de Alvos Simples
}

\author{
S. J. S. Sant'Anna, J. C. da S. Lacava e D. Fernandes
}

\begin{abstract}
Resumo-Neste trabalho é apresentado um software para o cálculo da Seção Reta Radar (RCS) e da Resposta Polarimétrica de dipolos elétricos impressos em estruturas planares multicamadas. O software é de fácil utilização, pois é estruturado em interfaces gráficas com o usuário. $O$ cálculo da RCS é baseado no método dos momentos no domínio espectral.
\end{abstract}

Palavras-Chave-Espalhamento eletromagnético, método dos momentos, seção reta radar, resposta polarimétrica.

\begin{abstract}
The computation of the radar cross section (RCS) and the polarimetric response of electric dipoles printed in multilayer planar structures is addressed in this work. Software based on spectral moment methods and implemented in IDL language is presented. This software is user-friendly and easy to operate, since it is based on graphical interfaces.
\end{abstract}

Index Terms-Sacttering, moment method, radar cross section, polarimetric response.

\section{INTRODUÇÃO}

O sensoriamento remoto de alvos na superfície terrestre, na faixa de microondas, ganhou grande impulso com o desenvolvimento e uso dos radares polarimétricos de abertura sintética. Com as imagens polarimétricas intensificaram-se os estudos do espalhamento eletromagnético provocado por alvos naturais ou não. As características geométricas e eletromagnéticas de um alvo são os principais fatores que afetam o seu espalhamento.

A caracterização do espalhamento eletromagnético de alvos terrestres na faixa de microondas torna-se, portanto, uma área de grande interesse para a comunidade científica do sensoriamento remoto. Através do entendimento dos mecanismos de espalhamento de um alvo simples podem-se desenvolver modelos de espalhamento de alvos mais complexos, tais como floresta, área urbana, entre outros. Na modelagem, estes alvos complexos são, geralmente, considerados serem compostos por alvos com geometria mais simples e conhecida (esfera, dipolo, cilindro, etc), possuindo diferentes tamanhos, orientações, formas e propriedades elétricas.

Neste contexto, se insere este trabalho que apresenta um software implementado em linguagem IDL (Interactive Data Language) [1] para o estudo de algumas características eletromagnéticas de um elemento espalhador metálico simples. Este elemento espalhador está impresso em uma estrutura com múltiplas camadas dielétricas. A excitação da estrutura é realizada através de uma onda plana e uniforme, elipticamente polarizada e com incidência oblíqua. Para tanto, este trabalho foi organizado em seções. Na Seção II é apresentado o procedimento geral para o cálculo dos campos eletromagnéticos em estruturas contendo

S.J.S.Sant'Anna, Divisão de Processamento de Imagens, INPE, São José dos Campos, SP, e-mail: sidnei@dpi.inpe.br. J.C.S. Lacava e D. Fernandes, Laboratório de Antenas e Propagação - LAP, Instituto Tecnológico de Aeronáutica, São José dos Campos, SP, e-mails: \{lacava,david\}@ita.br. Este trabalho foi parcialmente financiado pela FINEP, projeto CAPTAER múltiplas camadas isotrópicas, que tenham como fonte a densidade superficial de corrente elétrica sobre o elemento espalhador. Os parâmetros de caracterização eletromagnética de um alvo que podem ser obtidos através do software implementado são definidos na Seção III. As interfaces do software implementado são descritas na Seção IV juntamente com suas funcionalidades e finalmente na Seção V são expostas as conclusões a cerca dos resultados obtidos.

\section{FORMULAÇÃO TEÓRICA}

O problema do espalhamento eletromagnético é abordado utilizando-se a técnica de onda completa no domínio espectral. Esta técnica é, usualmente, empregada nos trabalhos desenvolvidos no Laboratório de Antenas e Propagação (LAP) do Instituto Tecnológico de Aeronáutica na área de projetos e análise de antenas de microfita planas e com múltiplas camadas [2]. No entanto, esta técnica foi adaptada ao caso em que a estrutura é excitada através de ondas planas e uniformes [3].

A estrutura em análise é composta por quatro camadas dielétricas lineares, homogêneas e isotrópicas superpostas, cada uma possuindo permissividade elétrica $\left(\varepsilon_{n}\right)$ e permeabilidade magnética $\left(\mu_{n}\right)$ complexas, com $n \in\{0,1,2, g\}$. A geometria desta estrutura é mostrada na Fig. 1, onde todas as camadas são supostas ilimitadas ao longo das direções $x$ e $y$. De outro forma, as duas camadas exteriores, o espaço livre (camada superior) e o ground (camada inferior), possuem espessuras ilimitadas, enquanto as duas camadas confinadas possuem espessuras variáveis $\left(h_{1}\right.$ e $\left.h_{2}\right)$. Um elemento metálico, o qual atuará como espalhador, é impresso na interface entre a camada 2 e o espaço livre ( plano $z=d$ ).

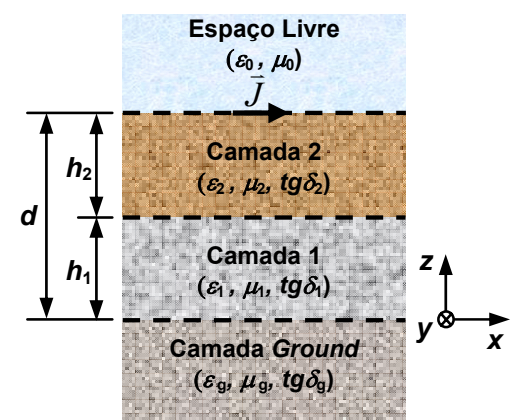

Fig. 1. Geometria da estrutura em análise (corte no plano $y=0$ ).

O elemento espalhador é caracterizado por uma superfície metálica retangular de dimensões $2 a$ e $2 b$, ao longo das direções $x$ e $y$, respectivamente. O retângulo é posicionado de tal forma que o seu centro geométrico coincida com o ponto de coordenadas $(0,0, d)$ de um sistema de coordenadas retangulares, ilustrado na Fig. 1. Este elemento pode ser particularizado para um dipolo elétrico planar orientado ao longo do eixo $x$, quando $2 a>2 b$. Uma onda plana e uniforme, elipticamente polarizada e com incidência oblíqua (definida pelos ângulos $\theta_{i}$ e $\phi_{i}$ de um sistema de 
coordenadas esféricas) é utilizada para excitar a estrutura. Esta onda induzirá uma densidade superficial de corrente elétrica sobre o elemento espalhador.

O procedimento para a determinação de expressões fechadas para os campos eletromagnéticos presentes em uma estrutura planar multicamadas e das respectivas funções de Green é descrito resumidamente no fluxograma da Fig. 2. De acordo com esta metodologia a estrutura é tratada como um problema de contorno e a corrente induzida na superfície metálica será a fonte virtual dos campos espalhados.

Partindo das equações de onda para cada camada, no domínio de Fourier, obtém-se um sistema de equações diferenciais. A partir deste sistema e após a aplicação das condições de contorno apropriadas em cada interface produz-se um sistema linear nas amplitudes dos campos eletromagnéticos transformados, possuindo $4 N+4$ equações e o mesmo número de incógnitas. A variável $N$ é igual ao número de camadas confinadas da estrutura, portanto, $N=2$ no caso da estrutura em análise. As funções Green espectrais para cada camada podem ser obtidas analiticamente resolvendo-se este sistema linear. Como exemplo, a expressão da função de Green espectral elétrica, que relaciona a componente do campo elétrico transformado na direção $x$ à componente $x$ da densidade superficial de corrente elétrica também na direção $x$ localizada na interface do plano $z=d$, é :

$G_{x x}^{(0)}\left(k_{x}, k_{y}, z\right)=-\frac{4 \omega^{2}}{u^{2}}\left(\frac{k_{x}^{2} \gamma_{0} \Omega_{0}^{e}}{\Delta_{e}}+\frac{k_{y}^{2} \omega \mu_{0} \Omega_{0}^{h}}{\Delta_{m}}\right) e^{-i \gamma_{0} z}$

onde

$$
\begin{aligned}
& \Omega_{0}^{e}=\gamma_{2}\left\{\varepsilon_{1} \gamma_{2}\left(\varepsilon_{1} \gamma_{g} \operatorname{sen} \theta_{1}-i \varepsilon_{g} \gamma_{1} \cos \theta_{1}\right) \operatorname{sen} \theta-\right. \\
& \left.\varepsilon_{2} \gamma_{1}\left(\varepsilon_{1} \gamma_{g} \cos \theta_{1}+i \varepsilon_{g} \gamma_{1} \operatorname{sen} \theta_{1}\right) \cos \theta\right\}, \\
& \Omega_{0}^{h}=\omega \mu_{2}\left\{\mu_{1} \gamma_{2}\left(\mu_{g} \gamma_{1} \cos \theta_{1}+i \mu_{1} \gamma_{g} \operatorname{sen} \theta_{1}\right) \cos \theta-\right. \\
& \left.\mu_{2} \gamma_{1}\left(\mu_{g} \gamma_{1} \operatorname{sen} \theta_{1}-i \mu_{1} \gamma_{g} \cos \theta_{1}\right) \operatorname{sen} \theta\right\} \\
& \Delta_{e}=-4 \omega^{3} e^{-i \theta_{0}}\left\{\varepsilon_{1} \gamma_{2}\left(\varepsilon_{2} \gamma_{0} \cos \theta+i \varepsilon_{0} \gamma_{2} \operatorname{sen} \theta\right)\right. \\
& \left(\varepsilon_{g} \gamma_{1} \cos \theta_{1}+i \varepsilon_{1} \gamma_{g} \operatorname{sen} \theta_{1}\right)+\varepsilon_{2} \gamma_{1}\left(\varepsilon_{0} \gamma_{2} \cos \theta+\right. \\
& \left.\left.i \varepsilon_{2} \gamma_{0} \operatorname{sen} \theta\right)\left(\varepsilon_{1} \gamma_{g} \cos \theta_{1}+i \varepsilon_{g} \gamma_{1} \operatorname{sen} \theta_{1}\right)\right\} \\
& \Delta_{m}=4 \omega^{3} e^{-i \theta_{0}}\left\{\mu_{1} \gamma_{2}\left(\mu_{2} \gamma_{0} \cos \theta+i \mu_{0} \gamma_{2} \operatorname{sen} \theta\right)\right. \\
& \left(\mu_{g} \gamma_{1} \cos \theta_{1}+i \mu_{1} \gamma_{g} \operatorname{sen} \theta_{1}\right)+\mu_{2} \gamma_{1}\left(\mu_{0} \gamma_{2} \cos \theta+\right. \\
& \left.\left.i \mu_{2} \gamma_{0} \operatorname{sen} \theta\right)\left(\mu_{1} \gamma_{g} \cos \theta_{1}+i \mu_{g} \gamma_{1} \operatorname{sen} \theta_{1}\right)\right\}, \\
& \gamma_{n}=(-1)^{\tau} \sqrt{k_{n}^{2}-u^{2}} \quad \operatorname{Im}\left\{\gamma_{n}\right\} \leq 0, \\
& u^{2}=k_{x}^{2}+k_{y}^{2}, \theta_{0}=\gamma_{0} d, \theta_{1}=\gamma_{1} h_{1}, \theta_{2}=\gamma_{2} d, \theta_{3}=\gamma_{2} h_{1} \text { e } \theta=\theta_{2}-\theta_{3} .
\end{aligned}
$$

Através da combinação das funções de Green espectrais e do campo excitador da estrutura, o qual está diretamente relacionado aos campos da onda incidente, monta-se um sistema de equações integrais. Neste sistema as incógnitas serão as componentes da densidade superficial de corrente elétrica $\boldsymbol{J}(x, y)$ induzida sobre a superfície condutora. A expressão para a componente na direção $x$ do campo excitador é dada por:

$$
\begin{gathered}
E_{0 x}^{s}(x, y, d)=-e^{i \Psi_{0}}\left\{\operatorname{sen} \phi_{i}\left(e^{i \Omega_{02}}+\Gamma_{h} e^{-i \Omega_{02}}\right) E_{i 0 h}+\right. \\
\left.\cos \theta_{i 0} \cos \phi_{i}\left(e^{i \Omega_{02}}-\Gamma_{v} e^{-i \Omega_{02}}\right) E_{i 0 v}\right\}
\end{gathered}
$$

onde $\Omega_{02}=k_{0} d_{2} \cos \theta_{i 0}, \Psi_{0}=k_{0} \operatorname{sen} \theta_{i 0}\left(x \cos \phi_{i}+y \operatorname{sen} \phi_{i}\right)$, e $\Gamma_{h}=E_{r 0 h} / E_{i 0 h}$ e $\Gamma_{v}=E_{r 0 v} / E_{i 0 v}$ são, respectivamente, os coeficientes de reflexão para as polarizações linear horizontal e vertical na interface $z=d$. Os coeficientes de reflexão são dados pela relação entre as amplitudes complexas dos campos elétricos incidente e refletido no espaço livre. Note que quantidades vetoriais são designadas por letras em negrito.

Para resolver o sistema de equação integral aplica-se o método dos momentos (MoM) no domínio espectral. Primeiramente, expande-se linearmente $\boldsymbol{J}(x, y)$ em funções de base, utiliza-se o método de Galerkin (onde as funções de teste e de base são definidas iguais) e o teorema de Parseval. Após estes passos a equação integral referente a $E_{0 x}^{S}$, é escrita:

$$
\begin{aligned}
& 4 \pi^{2} \iint_{\mathrm{S}} E_{0 x}^{S}(x, y, d) J_{p}^{*}(x, y) d x d y= \\
& \sum_{m=1}^{M} \sum_{n=1}^{N} I_{x m n} \int_{-\infty}^{+\infty} \int_{-\infty}^{+\infty} G_{x x}^{(0)} j_{m} j_{p}^{*} d k_{x} d k_{y},
\end{aligned}
$$

onde $S$ representa a superfície condutora, $J_{p}^{*}(x, y)$ representa o complexo conjugado da função de teste no domínio espacial, $I_{x m n}$ são os coeficientes complexos a serem determinados, $j_{m}\left(k_{x}, k_{y}\right)$ é a transformada de Fourier da função de base e $j_{p}^{*}\left(k_{x}, k_{y}\right)$ representa o complexo conjugado da transformada de Fourier da função de teste. Os índices $m$ e $p$ variam de 1 a $M$, enquanto que as variáveis espectrais $k_{x}$ e $k_{y}$ variam de $-\infty$ até $+\infty$. O sistema (8) pode ser representado na forma matricial $[V]=[Z] .[I]$, através das matrizes de excitação, de impedância e de coeficientes, respectivamente.

A solução do sistema linear de equações (8) ainda pode ser facilitada empregando-se algumas simplificações matemáticas. As simplificações adotadas são: a redução ao primeiro quadrante (estudo de paridade das funções de Green), a mudança de variáveis (coordenadas retangulares para coordenadas polares) e subtração do termo dominante (acelera a convergência da integração) [2]. Após estas simplificações a matriz [Z] fica representada por:

$$
[Z]=\frac{1}{\pi^{2}} \int_{\beta=0}^{+\infty} \int_{\alpha=0}^{\pi / 2}\left[A_{21} \cos ^{2} \alpha+A_{22} \operatorname{sen}^{2} \alpha\right] \mathbb{R}_{p m} d \alpha d \beta+A_{23}
$$

onde

$$
\begin{aligned}
& A_{20}=\frac{\beta^{2}}{\varepsilon_{0}+\varepsilon_{2}}, \\
& A_{21}=\frac{4 \omega^{2} \beta \gamma_{0} \Omega_{0}^{e}}{\Delta_{e}}-\frac{i A_{20}}{\omega},
\end{aligned}
$$




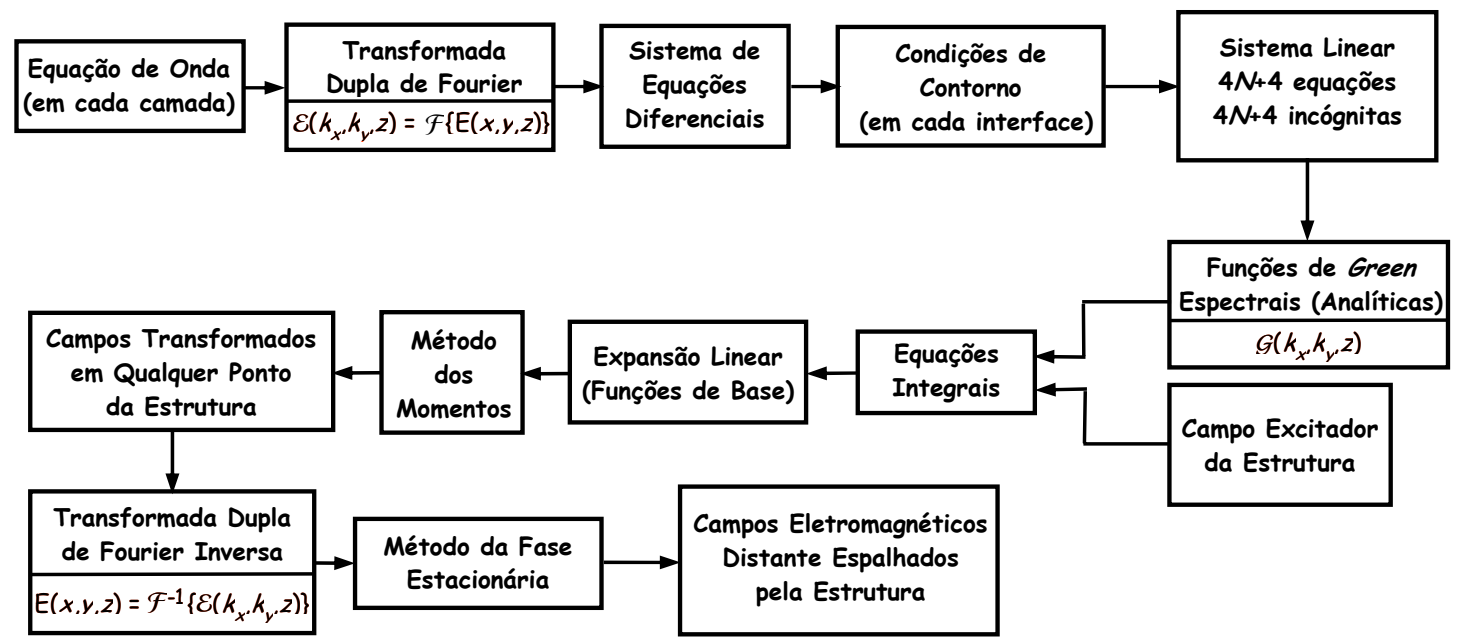

Fig. 2. Diagrama de blocos da metodologia.

$$
\begin{aligned}
& A_{22}=\frac{4 \omega^{3} \beta \mu_{0} \Omega_{0}^{h}}{\Delta_{m}}, \\
& A_{23}=\frac{i}{\omega \pi^{2}} \int_{0}^{+\infty} \int_{0}^{\pi / 2} A_{20} \cos ^{2} \alpha \mathbb{R}_{p m} d \alpha d \beta, \\
& \mathbb{R}_{p m}=\pi^{2} b^{2} J_{0}^{2}\left(b k_{y}\right) \operatorname{sinc}^{4}\left(k_{x} \Delta x / 2\right) \cos \left[k_{x}\left(x_{m}-x_{p}\right)\right],
\end{aligned}
$$

onde $\operatorname{sinc}(\alpha)=\operatorname{sen} \alpha / \alpha, J_{0}($.$) denota a função de Bessel de$ primeira espécie e ordem zero, $\Delta x=2 a /(M+1)$ e o termo $\boldsymbol{R}_{p m}$ é dado no caso da função de base triangular com efeitos de bordas.

Em(9) as integrais são conhecidas como integrais de Sommerfeld, que são extremamente oscilatórias e apresentam problemas de convergência. O integrando de $A_{23}$ é independente da freqüência de operação, enquanto o outro integrando depende da freqüência. Estes integrandos são funções oscilatórias na variável $\beta$ e as oscilações se agravam com o aumento do número de modos de expansão. Além do comportamento oscilatório o integrando do termo que é dependente da freqüência apresenta singularidades (pólos complexo) que estão associadas às ondas guiadas nas camadas. Estes dois fatos aliados à integração na variável $\beta$ ser indefinida (limite superior infinito), dificultam a realização de uma integração numérica com boa precisão. Portanto, é necessário se empregar estratégias para a realização das integrais numéricas quando são efetuadas sobre o eixo real.

Neste ponto os campos eletromagnéticos transformados em qualquer ponto da estrutura multicamadas podem ser determinados. Finalmente, os campos eletromagnéticos no domínio espacial são obtidos a partir da transformada inversa dupla de Fourier. O método da fase estacionária [4] é usado para se obter expressões assintóticas para os campos eletromagnéticos espalhados pela estrutura. O campo elétrico distante nos pontos de fase estacionária $k_{x e}=k_{0} \operatorname{sen} \theta \cos \phi$ e $k_{y e}=k_{0} \operatorname{sen} \theta \operatorname{sen} \phi \quad$ é então expresso por:

$$
\boldsymbol{E}_{0}(r, \theta, \phi) \cong-\frac{i k_{0}}{2 \pi} \frac{e^{-i k_{0} r}}{r} \cot \theta\left\{\hat{\boldsymbol{\theta}} \mathfrak{e}_{0 z}-\hat{\boldsymbol{\phi}} \eta_{0} \mathfrak{h}_{0 z}\right\},
$$

num sistema de coordenadas esféricas, onde $\eta_{0}$ é a impedância intrínseca do espaço livre, $k_{0}$ é o número de onda, $r$ é a distância entre a antena receptora e o alvo e as componentes longitudinais dos campos eletromagnéticos transformados $\left(\mathbb{e}_{0 z}\right.$ e $\left.\mathfrak{h}_{0 z}\right)$ são funções das variáveis $k_{x e}$ e $k_{y e}$. Vale observar que a partir dessa expressão assintótica e do conhecimento da densidade superficial de corrente, os elementos da matriz de espalhamento da estrutura são completamente determinados para qualquer direção de espalhamento e incidência.

\section{PARÂMETROS do ESPALHAMENTO}

A Seção Transversal Radar ou Seção Reta Radar (Radar Cross Section - RCS) é uma medida obtida a campo distante utilizada para caracterizar as propriedades de espalhamento de um alvo e possui unidade de área $\left(\mathrm{m}^{2}\right)$. Em geral, a RCS de um alvo é função da polarização da onda eletromagnética incidente, do ângulo de incidência, do ângulo de observação, da geometria e das propriedades elétricas do alvo e da freqüência de operação. A RCS de um objeto em uma dada direção é definida em [5], como sendo a seção transversal de um espalhador isotrópico que gera a mesma densidade de potência espalhada que o objeto na direção de observação. A RCS de um objeto (alvo) é definida por:

$$
\operatorname{RCS}\left(\boldsymbol{k}_{S}, \boldsymbol{k}_{i}\right)=\lim _{r \rightarrow \infty}\left[4 \pi r^{2} \frac{\left|\boldsymbol{E}^{S}\left(\boldsymbol{k}_{S}\right)\right|^{2}}{\left|\boldsymbol{E}^{i}\left(\boldsymbol{k}_{i}\right)\right|^{2}}\right],
$$

onde $\boldsymbol{E}^{i}\left(\boldsymbol{k}_{i}\right)$ é o vetor campo elétrico da onda plana incidente na direção de $\boldsymbol{k}_{i}$ e $\boldsymbol{E}^{S}\left(\boldsymbol{k}_{s}\right)$ é o vetor campo elétrico da onda espalhada na direção de $\boldsymbol{k}_{s}$ recebido a uma distância $r$ do alvo. A RCS também pode ser representada graficamente em função dos ângulos de elipsidade e de orientação da onda eletromagnética transmitida. De acordo com van Zyl ([6]) esta representação é denominada de resposta polarimétrica, a qual pode ser apresentada em co-polarização (polarização principal) ou em polarização cruzada. No primeiro caso as antenas transmissora e receptora possuem a mesma polarização e no segundo caso possuem polarizações ortogonais. 


\section{O SOFTWARE}

O software para o cálculo da RCS de estruturas planares multicamadas é denominado Four Layers Electromagnetic Scattering, e foi totalmente implementado em linguagem IDL. O software é totalmente amigável e de fácil utilização, já que foi estruturado em interfaces gráficas com o usuário. A interface principal do software é apresentada na Fig. 3a, dando os devidos créditos às instituições (Fig. 3b) de afiliação dos autores, assim como informações simplificadas (Fig. 3c) a respeito do software. A implementação do software foi realizada de tal forma que os dados de entrada foram relacionados a três grupos primários denominados por: parâmetros de entrada (Input Parameters), substratos (Substrates) e elemento espalhador (Scatter). Os dados de saída podem ser visualizados através do grupo de interfaces: da seção reta radar (RCS), da resposta polarimétrica ( $P O I$. Response) e da densidade de corrente (Density).

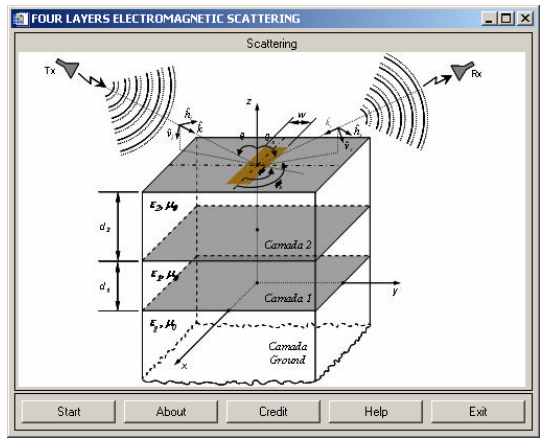

(a)

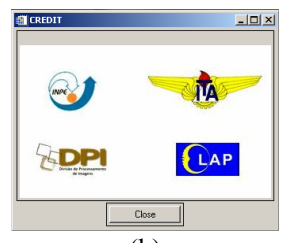

(b)

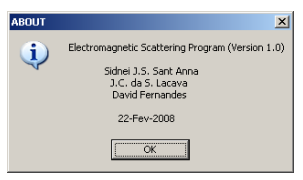

(c)
Fig. 3. Interfaces do software: (a) principal; (b) créditos e (c) informações.

$\mathrm{Na}$ interface do grupo parâmetros de entrada o usuário necessita fornecer a freqüência de operação, a direção de incidência da onda plana sobre a estrutura, o tipo de polarização desta onda e a direção de espalhamento. A freqüência de operação pode ser fornecida em $\mathrm{Hz}, \mathrm{kHz}, \mathrm{MHz}$ ou $\mathrm{GHz}$. A direção de incidência da onda plana é expressa através dos ângulos $\theta_{i}$ e $\phi_{i}$ em coordenadas esféricas, cuja unidade é o grau decimal. As polarizações elíptica e lineares, horizontal ou vertical, podem ser selecionadas, e após esta seleção o usuário deve digitar o valor complexo da amplitude do campo elétrico da onda incidente. Este valor complexo pode se fornecido em módulo e fase ou em partes real e imaginária, na unidade $V / m$. Os ângulos $\theta_{s}$ e $\phi_{s}$, também em coordenadas esféricas, fornecidos em graus determinam a direção do campo elétrico espalhado pela estrutura. Todos os parâmetros de entrada desta interface podem ser importados para seus respectivos campos a partir de um arquivo escrito no formato ascii de extensão .inp, mostrado na Fig. 4 no campo Input Files.

As características de cada camada dielétrica devem ser especificadas na interface do grupo substratos. As unidades $\mathrm{mm}$, $\mathrm{cm}, \mathrm{dm}, \mathrm{m}$ e polegadas podem ser usadas para definir a espessuras das camadas confinadas. A permeabilidade magnética e a permissividade elétrica das camadas são valores complexos que podem ser fornecidos em partes real e imaginária ou em seus respectivos valores relativos juntamente com a tangente de perdas. As características das camadas também podem ser importadas para seus respectivos campos a partir de um arquivo escrito no formato ascii de extensão . lay. Situação esta, que é ilustrada na Fig. 5 no campo denominado Layer Files.

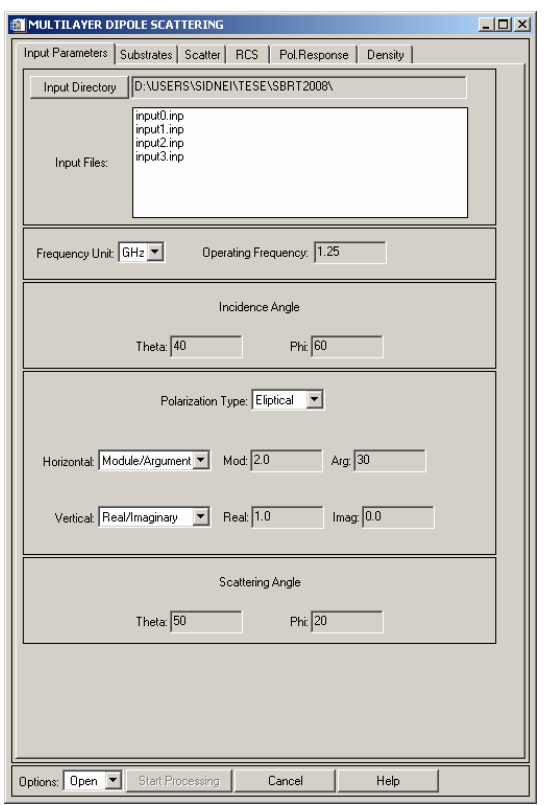

Fig. 4. Interface referente aos parâmetro de entrada.

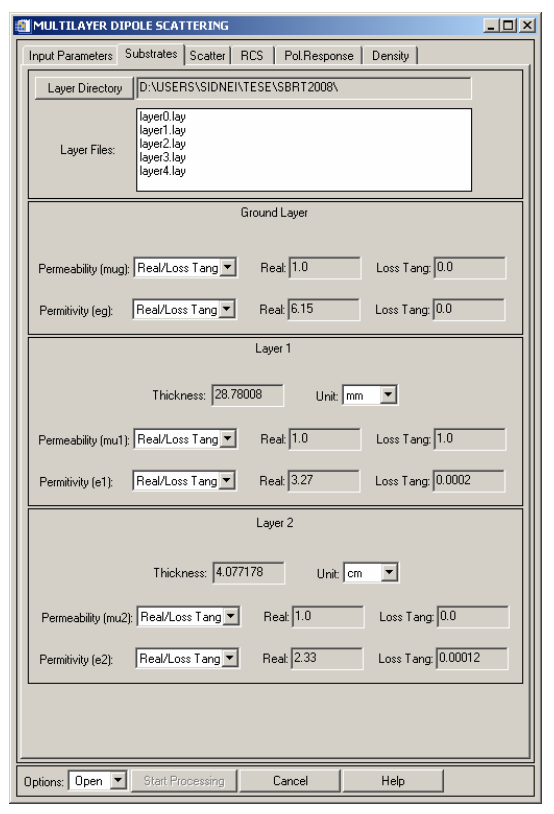

Fig. 5. Interface referente às camadas dielétricas.

$\mathrm{Na}$ interface do grupo elemento espalhador (Fig. 6) o usuário definirá as dimensões do elemento espalhador e também alguns parâmetros usados no método dos momentos. As unidades possíveis de serem usadas para as dimensões do dipolo são idênticas àquelas da espessura do substrato. Nos parâmetros do MoM o usuário pode selecionar funções de base de subdomínio triangular (rooftop) ou retangular, o numero de modos que a densidade superficial de corrente elétrica será expandida, se o efeito de bordas será ou não levado em consideração e também especificar as particularidades computacionais das integrais duplas que especificam a submatriz $[Z]$, definida em (9).

A integração na variável $\beta$ pode ser realizada através de duas 
estratégias distintas: ao longo do eixo real ou de um contorno parabólico (Fig. 7). A estratégia de integração é definida no campo Integral Path e caso o contorno parabólico seja selecionado, o usuário deve fornecer os valores dos parâmetros $A$ e $B$, como mostrado na Fig. 7. Geralmente, os valores atribuídos a $A$ e $B$ são, respectivamente, da ordem de $0,1 k_{0}$ e $1,1 k_{0} \varepsilon_{r m}$, onde $\varepsilon_{r m}=\left(\operatorname{Max}\left\{\varepsilon_{r l}, \varepsilon_{r 2}\right\}\right)^{1 / 2}$. O contorno parabólico é indicado para estruturas nas quais as espessuras das camadas confinadas sejam maiores que um comprimento de onda da freqüência de operação, pois o número pólos aumenta com o aumento da espessura destas camadas. Na primeira estratégia, são definidos os subintervalos de $\left[0, k_{0}\right],\left[k_{0}, k_{0} \varepsilon_{r m},\right]$ e $\left[k_{0} \varepsilon_{r m}, \infty\right)$ para se realizar a integração. Nesta implementação ainda é possível subdividir os intervalos de integração das variáveis $\alpha$ e $\beta$ em intervalos de tamanhos iguais. Na integração da variável $\beta$ do termo dependente da freqüência esta subdivisão é realizada em cada um dos três intervalos anteriormente definidos.

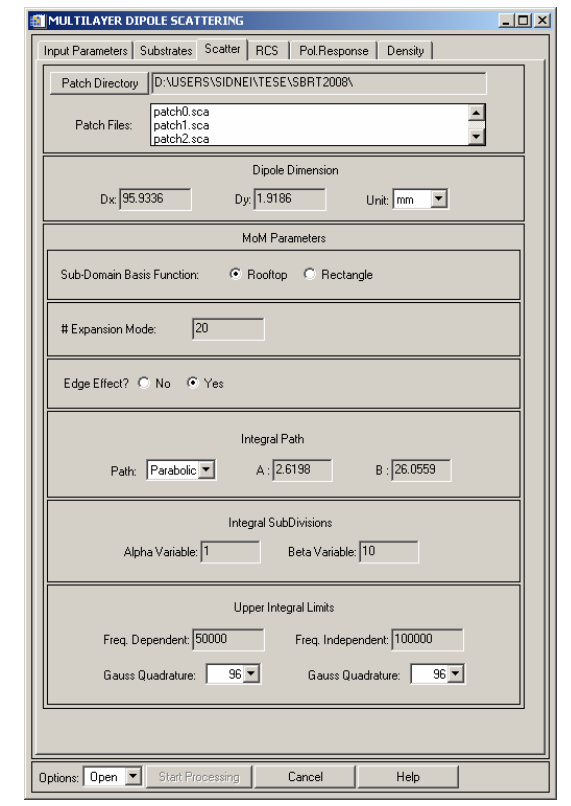

Fig. 6. Interface referente ao elemento espalhador.

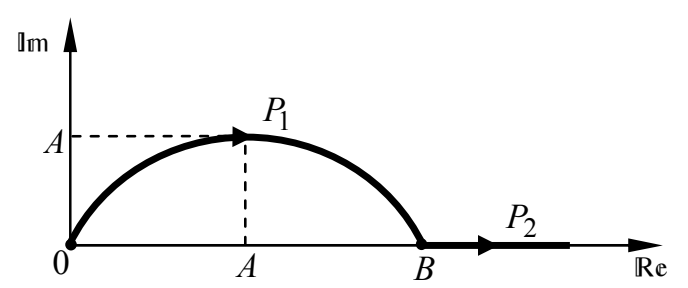

Fig. 7. Contorno Parabólico.

O número de intervalos de tamanhos iguais das subdivisões das variáveis $\alpha$ e $\beta$ devem ser fornecidos nos campos definidos por Integral Subdivisions (Fig. 6). Já nos campos denominados por Upper Integral Limits devem ser determinados os limites superiores das integrais (na variável $\beta$ ) dos termos dependente e independente da freqüência, bem como o número de pontos da transformação da quadratura de Gauss para cada uma destas integrais. A rotina implementada para calcular as integrais duplas usa a regra de quadratura de Gauss-Legendre, podendo-se utilizar $6,8,10,16,20,32,48,64$ ou 96 pontos de transformação.
Da mesma forma que nas interfaces dos outros dois grupos, também é possível importar todos os dados referentes a esta interface de um arquivo escrito no formato ascii. Este arquivo deve ter extensão . sca. Cabe ressaltar que neste software o botão chamado de Start Processing somente ficará ativo após todos os campos dos três grupos de interfaces dos dados de entrada serem devidamente preenchidos.

Os gráficos ilustrados nas interfaces de saída são referentes a um dipolo elétrico excitado por uma onda plana verticalmente polarizada, cuja amplitude do campo elétrico é $1 \mathrm{~V} / \mathrm{m}$. Os valores atribuídos a cada parâmetro são apresentados nos campos das interfaces (Figs. 4 a 6), excetuando-se o campo da polarização da onda incidente (Fig. 4), no qual são ilustradas as amplitudes do campo elétrico de uma onda elipticamente polarizada.

As curvas de RCS tanto em elevação $\left(\theta_{s}\right.$ variando de $-90^{\circ}$ a $+90^{\circ}$ e $\phi_{s}$ fixo), ilustrada na Fig. 8, quanto em azimute ( $\theta_{s}$ fixo e $\phi_{s}$ variando de $-180^{\circ} \mathrm{a}+180^{\circ}$ ), mostrada na Fig. 9, podem ser visualizadas em escala linear $\left(\mathrm{m}^{2}\right)$ na interface de saída dos dados. Nestas figuras observa-se que os valores de RCS são muito baixo, o que é decorrente das dimensões milimétricas do dipolo elétrico impresso utilizados nos cálculos. A visualização dos gráficos pode ser feita apenas de uma curva (proveniente da execução do software) ou de várias curvas simultaneamente (processo de importação de vários arquivos). Os arquivos de dados a serem importados devem estar escritos no formato ascii e possuir extensão .azm (azimute) ou .elv (elevação). Os campos Angle_Min e Angle_Max são utilizados para traçar gráficos no intervalo de valores definidos por estes parâmetros (Zoom).

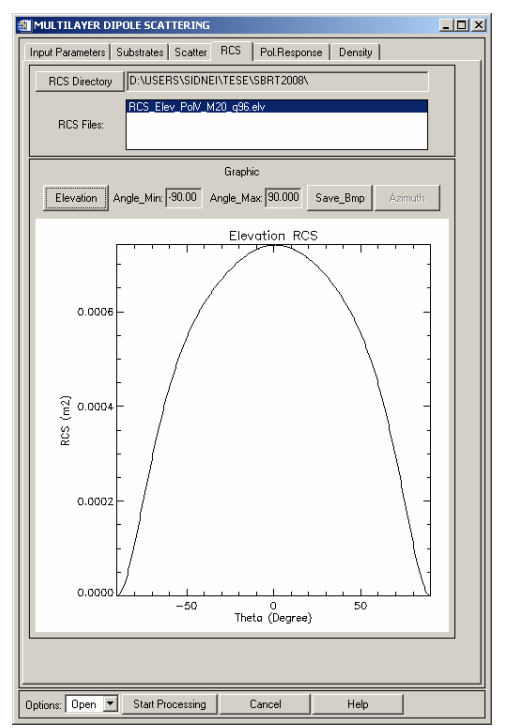

Fig. 8. Interface de saída da RCS em elevação.

No cálculo da resposta polarimétrica é levado em consideração o teorema da reciprocidade, neste caso a polarização da onda de incidência deve ser linear vertical ou horizontal. Os gráficos (tridimensionais) da resposta polarimétrica em co-polarização e em polarização cruzada são ilustradas, respectivamente nas Figs. 10 e 11. Estes gráficos somente podem ser visualizados um por vez, seja oriundo da execução do software ou importado de arquivos. Os arquivos de dados a serem importados devem estar escritos no formato ascii e com extensão .cop (co-polarização) ou .crp (polarização cruzada). 


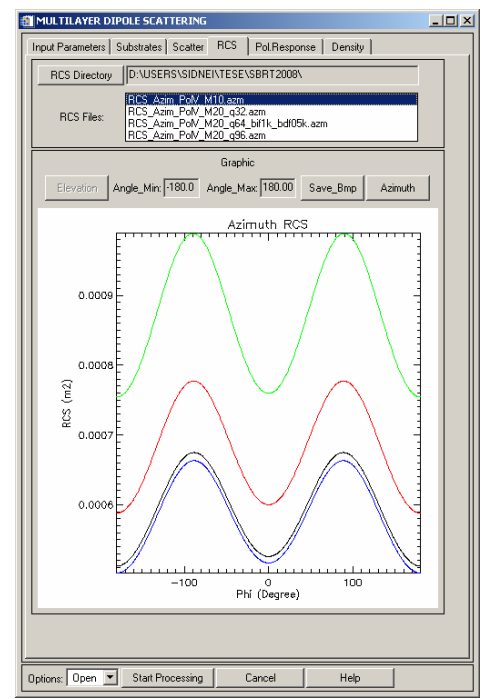

Fig. 9. Interface de saída da RCS em azimute.

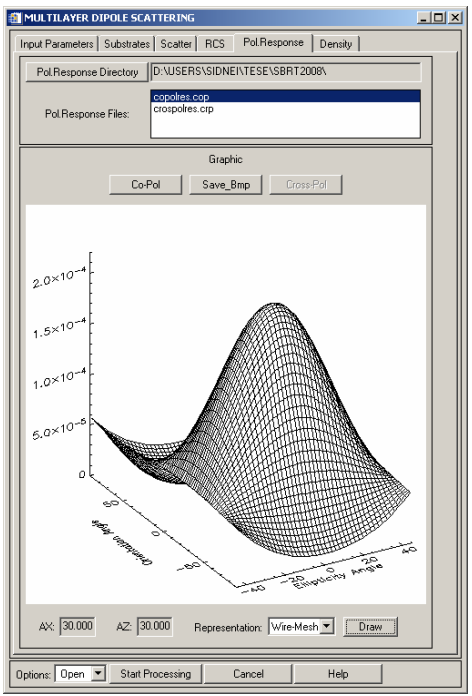

Fig. 10. Interface de saída da resposta polarimétrica em co-polarização.

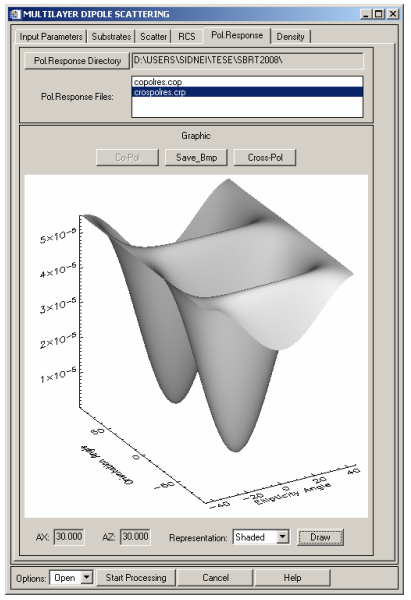

(a)

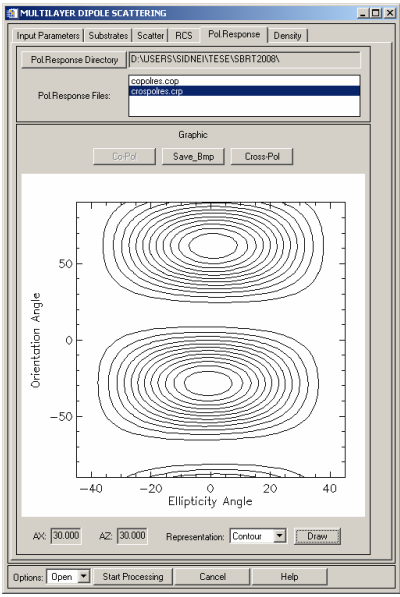

(b)
Fig. 11. Interface de saída da resposta polarimétrica em polarização cruzada: (a) sombreada e (b) curvas de nível.

A partir das Figs. 10 e 11 ainda é possível observar três tipos de representação distintos para a resposta polarimétrica (aramado, sombreado e de curvas de nível). Na interface de saída da resposta polarimétrica pode-se definir a direção de visualização dos gráficos através dos campos $A X$ e $A Z$. O botão Draw é utilizado para desenhar um gráfico após ser realizada uma mudança no mesmo, seja da direção de visualização, sua representação ou do seu tipo.

Os gráficos tridimensionais do módulo e da fase da densidade superficial de corrente induzida no elemento espalhador são mostrados, respectivamente na Fig. 12a e 12b. Nesta interface se encontram as mesmas funcionalidades encontradas na interface da resposta polarimétrica. No entanto os arquivos de dados a serem importados devem estar escritos no formato ascii e com extensão . amp (módulo) ou .pha (fase).

Todos os gráficos visualizados neste software podem ser salvos em um arquivo no formato bitmap.

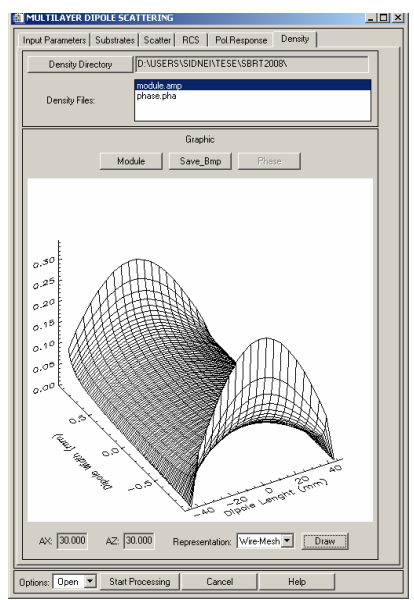

(a)

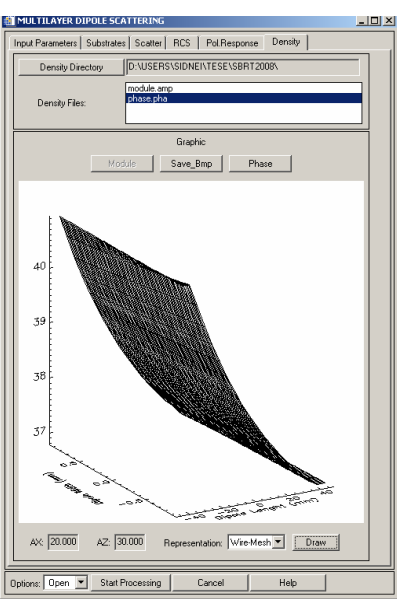

(b)
Fig. 12. Interface de saída da densidade de corrente: (a) módulo e (b) fase.

\section{CONCLUSÕES}

Neste trabalho é apresentado um software para o cálculo da RCS de dipolos elétricos impressos em estruturas planares multicamadas, denominado Four Layers Electromagnetic Scattering. O desenvolvimento teórico para a determinação da RCS é baseado no MoM no domínio espectral. O software é desenvolvido totalmente na plataforma IDL a qual tem se mostrado uma ótima ferramenta de cálculo e análise numérica, além de possuir uma grande capacidade gráfica. O software permite que o usuário analise várias configurações de parâmetros de entrada, camadas dielétricas e particularidades do MoM. Os resultados apresentados pelo software estão de acordo com os resultados fornecidos pelo software comercial IE3D ${ }^{\mathrm{TM}}$.

\section{REFERÊNCIAS}

[1] Interactive Data Language (IDL), Version 6.0.1, Research System, 2003.

[2] I. Bianchi, "Análise de antenas de microfita com substratos anisotrópicos usando computação simbólica e método dos momentos," Tese de Doutorado. ITA, São José dos Campos, 2006.

[3] S.J.S. Sant'Anna, "Caracterização do espalhamento eletromagnético de alvos simples em estruturas multicamadas," Exame de Qualificação. ITA, São José dos Campos, 2007.

[4] R.E. Collin e F.J. Zucker, Antenna Theory: Part 1, McGraw-Hill Book Company, New York, 1969.

[5] A.K. Fung, Microwave scattering and emission models and their applicatioins. Norwood, Artech House, 1994, 573p.

[6] J.J. van Zyl "Unsupervised classification of scattering behavior using radar polarimetry data," IEEE Trans. Geosci. Remote Sensing, v. 27, n. 1, pp. 36-45, 1989. 